\title{
ARRAY-COMPARATIVE GENOMIC HYBRIDIZATION RESULTS IN CLINICALLY AFFECTED CASES WITH APPARENTLY BALANCED CHROMOSOMAL REARRANGEMENTS
}

Satkin NB*, Karaman B, Ergin S, Kayserili H, Kalelioglu IH, Has R, Yuksel A, Basaran S

*Corresponding Author: Nihan B. Satkin, Ph.D., Department of Medical Genetics, Istanbul University Faculty of Medicine, Millete Street, 34093, Istanbul, Turkey. Tel.: +90-536-561-0313. Fax:+90-212-414-2000. E-mail: bilgenihan@gmail.com

\begin{abstract}
Carriers of apparently balanced chromosomal rearrangements (ABCRs) have a 2-3-fold higher risk of carrying an abnormal phenotype, when compared to the average population. Apparently balanced chromosomal rearrangements can be imbalanced at the submicroscopic level, and changes in the gene structure, formation of a new chimeric gene, gain or loss of function of the genes and altered imprinting pattern may also affect the phenotype. Chromosomal microarray (CMA) is an efficient tool to detect submicroscopic imbalances at the breakpoints as well as in the whole genome. We aimed to determine the effectiveness of array-comparative genomic hybridization (aCGH) application in phenotypically affected cases with ABCRs at a single center from Turkey. Thirty-four affected cases (13 prenatal, 21 postnatal) carrying ABCRs were investigated with CMA. In postnatal series, ABCRs were familial in 7 and de novo in 14 cases. Seven de novo cases were imbalanced (in postnatal series $33.3 \%$ and in de novo cases $50.0 \%$ ). Out of 13 prenatal cases, five were familial and eight were de novo in origin and two de novo cases were imbalanced (in $15.4 \%$ prenatal series and in $25.0 \%$ de novo cases). No cryptic imbalance was observed in familial cases. The anomaly rates with array studies ranged between 14.3-25.0\% in familial and between 20.0-57.5\% in de novo cases of postnatal series in the literature. Studies focused on prenatal ABCR cases with abnormal ultrasound findings are limited and no submicroscopic imbalance was reported in the cohorts. When de novo postnatal or prenatal results were combined, the percentage of abnormalities
\end{abstract}

Department of Medical Genetics, Istanbul Faculty of Medicine, Istanbul, Turkey detected by CMA was $40.9 \%$. Taking this contribution into consideration, all ABCRs should be investigated by CMA even if the fetal ultrasound findings are normal.

Keywords: Apparently balanced structural chromosomal abnormalities (ABCRs); Array-comparative genomic hybridization (aCGH); Chromosomal microarray (CMA); Cryptic genomic imbalances, Fetal ultrasound findings.

\section{INTRODUCTION}

Balanced chromosomal rearrangements comprising translocations or inversions with an incidence $0.52 \%$ in the general population are structural chromosomal rearrangements without cytogenetically detected imbalances [1]. As conventional karyotyping might not be able to detect the smaller than 5-10 Mb imbalances, some submicroscopic imbalances can be overlooked [2]. These types of rearrangements are called as apparently balanced chromosomal rearrangements (ABCRs) and can be familial or de novo in origin. Warburton [3] determined the risk of phenotypical abnormalities as $6.1 \%$ for de novo ABCR translocations and inversions in a large prenatal series. Madan et al. [4] estimated the risk as $23.0 \%$ for complex chromosome rearrangements (CCRs), defining structural rearrangements involving more than two breakpoints. Especially, if the ABCR is de novo, it is important to search the submicroscopic genomic imbalances. Not only the cryptic imbalances could affect the phenotype but also other mechanisms, such as impairment of the expression of the gene in its new location (positional effects), disruption of genes at the breakpoints, formation of a new chimeric gene and disruption of the parental imprinting pattern might have a role [5].

The application of chromosomal microarray (CMA) has become an essential tool in routine diagnostics to detect 
the submicroscopic genomic imbalances for genome-wide screening at kilobase (kb) levels. Chromosomal microarray contributes a $15.0-20.0 \%$ detection rate of cryptic chromosomal imbalance in selected patients who have multiple congenital anomalies/intellectual disability (MCA/ID) with normal karyotypes [6,7].

We report here the CMA results of the 34 clinically affected patients (13 prenatal, 21 postnatal) carrying de novo or familial ABCRs of a single center in Turkey. The aim of the study was to add new cases to the literature, to present the rate of submicroscopic genomic imbalances according to different type of chromosomal abnormalities such as translocations, inversions, and CCRs, which are important in genetic counseling, and these findings were combined with published studies to provide the newest integrated insights.

\section{MATERIALS AND METHODS}

The study contains the cytogenetic and array-comparative genomic hybridization (aCGH) results of 34 affected patients (13 prenatal, 21 postnatal) carrying ABCRs and 76 of family members (including parents and siblings) investigated between the years 2001-2017. All cases were examined, counseled and laboratory work was performed at the Department of Medical Genetics, Istanbul University Faculty of Medicine, Istanbul, Turkey. Fetal ultrasonography (USG) and invasive procedures [one chorionic villus sampling (CVS), four fetal blood sampling (FSB) and eight by amniocentesis $(\mathrm{AC})]$ were performed at the Perinatology Division of the Obstetrics and Gynecology Department, Istanbul University Faculty of Medicine, Istanbul, Turkey.

Lymphocytes cultures of peripheral and cord blood samples, amniotic fluid and chorionic villus samples using short and long-term cell cultures were performed according to traditional techniques. Routine cytogenetic analyses were performed on metaphase chromosomes at 550-600 banding levels using Giemsa-Pancreatin-Leishman's banding.

Chromosomal Microarray Analysis. Genomic DNA was extracted using High Pure polymerase chain reaction (PCR) Template Preparation kit following the manufacturer's protocol (Roche Diagnostics, Indianapolis, IN, USA). Chromosomal microarray testing was performed in patients using NimbleGen $3 \times 1.4 \mathrm{M}$ Whole-Genome Tiling Array, which has $1,400,000$ copy number variations $(\mathrm{CNV})$ probes across the entire genome according to the manufacturer's instructions (Roche NimbleGen, Madison, WI, USA).

The SurePrint G3+SNP (single nucleotide polymorphism) Human CGH Microarray $(4 \times 180 \mathrm{~K})$ dual-color array containing more than 170,334 distinct biological probes with $13 \mathrm{~kb}$ genome-wide median probes spacing was carried out following the protocols provided by the manufacturer (Agilent Technologies Inc., Santa Clara, CA, USA) in parents to detect inheritance and for confirmation of detected CNVs that were larger than $1 \mathrm{Mb}$ in index cases.

Aberration calls using Nexus Copy Number (BioDiscovery, El Segundo, CA, USA) was implemented following thresholds for copy numbers; $100 \mathrm{~kb}$ gains, $50 \mathrm{~kb}$ losses across the genome with a minimum of 25 markers to make the call. For regions of known significance, the minimal setting was $25 \mathrm{~kb}$ and 25 markers for gains and losses. Detected variations were classified according to the American College of Medical Genetics and Genomics (ACMG) guidelines [8,9]. Only likely pathogenic or pathogenic variations were reported and uncertain $\mathrm{CNVs}$ were evaluated by taking into consideration of ACMG classification scores $[8,9]$.

This study was reviewed and approved by the Ethics Committee of the Istanbul Medical Faculty [N. 08/13]. Written informed consent was obtained from all subjects or their legal guardians included in this study.

\section{RESULTS}

Postnatal Cases. Postnatal cases $(n=21)$ consisted of four inversions, eight CCRs and nine translocations. Familial inheritance was found in seven cases (33.3\%) (three inversions, four translocations) and 14 cases were de novo in origin $(66.6 \%)$ (one inversion, five translocations and eight CCRs). Clinical findings, initial karyotypes, and CMA results of all cases are shown in Tables 1 and 2.

No cryptic genomic imbalance by CMA was observed in familial cases $(n=7)$. Of 14 de novo cases, seven presented with at least one imbalance $(50.0 \%)$. These CNVs were 12 deletions and one duplication, of which the size ranged from $1.1 \mathrm{Mb}$ to $10.6 \mathrm{Mb}$. Five imbalances were at breakpoints or near to breakpoints of the rearrangements (38.4\%), and eight were at different regions (61.5\%).

Of five de novo translocations, only one had a $4.8 \mathrm{Mb}$ duplication $(20.0 \%)$ at the breakpoint of the translocation (case 1) (Table 1). Chromosomal microarray revealed a deletion near to the breakpoint of a de novo inversion (case 10) (Table 1).

All CCRs $(n=8)$ were de novo and CMA revealed 11 deletions in five of them $(62.5 \%)$. Of 11 deletions, two were at breakpoints (cases 10 and 21), one was near to breakpoints (case 14) and eight were at different regions (cases 15, 16, 20, 21) (Table 1).

Prenatal Cases. Of 13 prenatal cases, nine were translocations (two familial and seven de novo in origin), three were inversions (all familial), and one was de novo CCR. An imbalance has been found in two of 13 cases (15.4\%) 
Table 1. Clinical findings, karyotype and chromosomal microarray results for the postnatal patients.

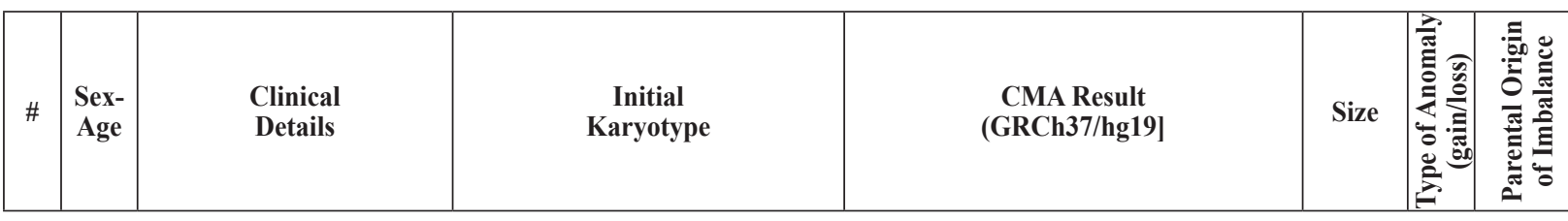

Translocations

\begin{tabular}{|c|c|c|c|c|c|c|c|}
\hline $1^{\mathrm{a}}$ & M-4 & $\begin{array}{l}\text { consanguineous marriage; } \\
\text { stereotypic hand } \\
\text { movements, PSMR; short } \\
\text { eye contact duration; } \\
\text { pathology in brain MRI; } \\
\text { ASD }\end{array}$ & $46, \mathrm{XY}, \mathrm{t}(4 ; 10)(\mathrm{q} 34.2 ; \mathrm{q} 26.2)$ & 10q26.3 (130669231-135534747)x3 & $4.8 \mathrm{Mb}$ & gain & de novo \\
\hline $2^{\mathrm{a}}$ & M-13 & $\begin{array}{l}\text { minimal nonspecific } \\
\text { ID; hypo-glycemia; } \\
\text { cryptorchidism; facial } \\
\text { dysmorphism; short neck; } \\
\text { hypo-myelinization and } \\
\text { subarachnoid enlargement } \\
\text { in cranial CT }\end{array}$ & $46, \mathrm{XY}, \mathrm{t}(4 ; 10)(\mathrm{q} 25 ; \mathrm{q} 22.1)$ & normal & - & - & - \\
\hline $3^{\mathrm{a}}$ & M-6 & $\begin{array}{l}\text { PSMR; anal stenosis; } \\
\text { bilateral inguinal hernia; } \\
\text { facial dysmorphism; } \\
\text { macrocephaly; short fin- } \\
\text { gers and clinodactyly on } \\
\text { the fifth finger }\end{array}$ & $46, \mathrm{XY}, \mathrm{t}(5 ; 17)(\mathrm{q} 12.2 ; \mathrm{q} 21.33)$ & normal & - & - & - \\
\hline $4^{\mathrm{a}}$ & M-4 & $\begin{array}{l}\text { PSMR; drug therapy } \\
\text { due to convulsion; facial } \\
\text { dysmorphism; pes planus }\end{array}$ & $46, \mathrm{XY}, \mathrm{t}(2 ; 10)(\mathrm{q} 33.2 ; \mathrm{p} 12.32)$ & normal & - & - & - \\
\hline $5^{\text {a }}$ & F-7 & $\begin{array}{l}\text { PSMR; hypotonia; } \\
\text { central obesity; childhood } \\
\text { polyphagia }\end{array}$ & $46, \mathrm{XX}, \mathrm{t}(6 ; 9)(\mathrm{q} 25.1 ; \mathrm{q} 32)$ & normal & - & - & - \\
\hline $6^{\mathrm{b}}$ & M-11 & $\begin{array}{l}\text { consanguineous marriage; } \\
\text { ID; hyperactivity; poor eye } \\
\text { contact, repetitive speech; } \\
\text { family history of MR } \\
\text { (paternal side); fragile X } \\
\text { test normal }\end{array}$ & $46, \mathrm{XY}, \mathrm{t}(9 ; 10)(\mathrm{p} 22 ; \mathrm{q} 24) \mathrm{pat}$ & normal & - & & - \\
\hline $7^{\mathrm{b}}$ & M-13 & $\begin{array}{l}\text { consanguineous marriage; } \\
\text { mild ID; brother and } \\
\text { maternal aunt's daughter } \\
\text { affected; microphthalmia; } \\
\text { severe PSMR; muscle } \\
\text { atrophy; microcephaly; } \\
\text { bilateral hydronephrosis; } \\
\text { facial dysmorphism }\end{array}$ & $46, \mathrm{XY}, \mathrm{t}(6 ; 19)(\mathrm{p} 11 ; \mathrm{p} 13.2) \mathrm{mat}$ & normal & - & - & - \\
\hline $8^{\mathrm{b}}$ & F-9 & $\begin{array}{l}\text { consanguineous marriage; } \\
\text { microcephaly; facial } \\
\text { dysmorphism; clinodactyly; } \\
\text { cranial CT consistent with } \\
\text { Lissencephaly type } 1\end{array}$ & $\begin{array}{l}46, \mathrm{XX}, \mathrm{t}(3 ; 4)(\mathrm{p} 22 ; \mathrm{q} 26) \mathrm{mat} \\
\text { (negative FISH result for Smith- } \\
\text { Magenis-Dieker Probe) }\end{array}$ & normal & - & - & - \\
\hline $9^{\mathrm{b}}$ & M-1 & $\begin{array}{l}\text { consanguineous marriage; } \\
\text { facial dysmorphism; CHD; } \\
\text { right kidney agenesis, } \\
\text { left kidney size increase; } \\
\text { undescended testicle; } \\
\text { anal atresia }\end{array}$ & $46, \mathrm{XY}, \mathrm{t}(8 ; 22)(\mathrm{p} 11.2 ; \mathrm{q} 22) \mathrm{mat}$ & normal & - & - & - \\
\hline
\end{tabular}

\begin{tabular}{|l|l|l|l|l|l|l|l|}
\hline \multicolumn{2}{|c|}{ Inversions } \\
\hline $10^{\mathrm{a}}$ & F-7 & $\begin{array}{l}\text { neuromotor retardation; } \\
\text { seizures; growth } \\
\text { retardation; microcephaly; } \\
\text { facial dysmorphism; } \\
\text { clinodactyly; pathology } \\
\text { in brain MRI }\end{array}$ & 46,XX,inv(1)(q25.1q32.2) & $1 \mathrm{q} 23.3 \mathrm{q} 24.2(161969134-169579696) \times 1$ & $7.6 \mathrm{Mb}$ & loss & de novo \\
\hline
\end{tabular}


Continuation of the previous page

\begin{tabular}{|c|c|c|c|c|c|c|c|}
\hline $11^{\mathrm{b}}$ & M-13 & $\begin{array}{l}\text { ID; undescended testicle; } \\
\text { advanced bone age; } \\
\text { obesity; agitation; fragile X } \\
\text { test normal }\end{array}$ & 46,XY,parinv(12)(p13.3q13.1)pat & normal & - & - & - \\
\hline $12^{b}$ & M-3 & $\begin{array}{l}\text { consanguineous marriage; } \\
\text { PSMR; growth retardation; } \\
\text { microcephaly; facial } \\
\text { dysmorphism; clinodactyly; } \\
\text { joint hypermobility }\end{array}$ & 46,XY,inv(12)(p11q14)mat & normal & - & - & - \\
\hline $13^{b}$ & M-5 & $\begin{array}{l}\text { consanguineous } \\
\text { marriage; pectus } \\
\text { excavates; pulmonary } \\
\text { stenosis; cryptorchidism, } \\
\text { micropenis; lytic bone } \\
\text { lesions; mutation negative } \\
\text { in RAF1, KRAS, SHOC2 } \\
\text { genes }\end{array}$ & 46,XY,parinv(12)(q21.2q24.1)mat & normal & - & - & - \\
\hline
\end{tabular}

Complex Chromosome Rearrangements

\begin{tabular}{|c|c|c|c|c|c|c|c|}
\hline $14^{\mathrm{a}}$ & M-4 & $\begin{array}{l}\text { PSMR; hypotonia; } \\
\text { microcephaly; syndactyly; } \\
\text { lack of eye contact }\end{array}$ & $\begin{array}{l}\text { 46,XY,t }(8 ; 13)(\mathrm{q} 24.13 ; \mathrm{q} 21.2), \\
\text { ins }(2)(\mathrm{p} 16.2 \mathrm{q} 33.2 \mathrm{q} 22.2)\end{array}$ & $2 q 36.1 q 36.3(225194399-228263782) \times 1$ & $3.0 \mathrm{Mb}$ & loss & de novo \\
\hline $15^{\mathrm{a}}$ & M-9 & $\begin{array}{l}\text { PSMR; alopecia; } \\
\text { hyperpigmentation; facial } \\
\text { dysmorphism; bilateral } \\
\text { cubitus valgus; accessory } \\
\text { nipple on bilateral nipple } \\
\text { line; tracheostomy }\end{array}$ & $\begin{array}{l}\text { 46,XY,inv }(3)(\mathrm{p} 13 \mathrm{p} 25) \mathrm{t}(11 ; 18) \\
(\mathrm{p} 13.5 ; \mathrm{q} 12.2)\end{array}$ & $\begin{array}{l}4 q 13.3(71013108-72174576) \times 1 \\
4 q 13.3 q 21.2(73373677-76306815) \times 1\end{array}$ & $\begin{array}{l}1.1 \mathrm{Mb} \\
2.9 \mathrm{Mb}\end{array}$ & $\begin{array}{l}\text { loss } \\
\text { loss }\end{array}$ & de novo \\
\hline $16^{\mathrm{a}}$ & M-1 & $\begin{array}{l}\text { bilateral aniridia; } \\
\text { cryptorchidism; } \\
\text { micropenis; heart murmur; } \\
\text { hypertonia of the lower } \\
\text { extremities }\end{array}$ & $\begin{array}{l}\text { 46,XY,t(3;15;21)(p13;q21.1; } \\
\text { q22.3),t(4;16)(q31;p31.1) }\end{array}$ & $11 \mathrm{p} 14.13(30031595-33045209) \times 1$ & $2.5 \mathrm{Mb}$ & loss & de novo \\
\hline $17^{\mathrm{a}}$ & F-3 & $\begin{array}{l}\text { PSMR; microcephaly; } \\
\text { dystrophinopathy; } \\
\text { myogenic EMG findings; } \\
\text { walking difficulties }\end{array}$ & $46, X, t(X ; 13 ; 17)(\mathrm{p} 21 ; \mathrm{q} 13 ; \mathrm{q} 22)$ & normal & - & - & - \\
\hline 18 & M-3 & $\begin{array}{l}\text { CHD; negative FISH result } \\
\text { for DiGeorge syndrome }\end{array}$ & $\begin{array}{l}\text { 46,XY,der(3)(15qter } \rightarrow 15 \mathrm{q} 22.3: \\
: 3 \mathrm{p} 11.2 \rightarrow 3 \mathrm{qter}) \operatorname{der}(15)(15 \mathrm{pter} \rightarrow \\
15 \mathrm{q} 22.3:: 3 \mathrm{p} 26 \rightarrow 3 \mathrm{p} 11.2: \\
: 3 \mathrm{p} 26 \rightarrow 3 \text { pter })\end{array}$ & normal & - & - & - \\
\hline $19^{a}$ & F-13 & $\begin{array}{l}\text { mild ID; dystrophinopathy; } \\
\text { Gowers' sign; pektus } \\
\text { ekskavatum; minimal facial } \\
\text { dysmorphism; normal } \\
\text { MLPA result }\end{array}$ & $\begin{array}{l}\text { 46,X,t(X;8;14)(8qter } \rightarrow \text { 8q11: } \\
: X p 21 \rightarrow \text { Xqter })(X p t e r \rightarrow X p 21: \\
: 8 p 21.1 \rightarrow 8 q 11:: 14 q 13)\end{array}$ & normal & - & - & - \\
\hline $20^{\mathrm{a}}$ & M-4 & $\begin{array}{l}\text { consanguineous } \\
\text { marriage; neuromotor } \\
\text { retardation; mild ID; facial } \\
\text { dysmorphism; brachy- } \\
\text { cephaly; hypoplastic } \\
\text { scrotum }\end{array}$ & $\begin{array}{l}\text { 46,XY,t(1;18)(q32.1;q23),t(5;12) } \\
(\mathrm{p} 14.2 ; \mathrm{q} 21.2)\end{array}$ & $\begin{array}{l}5 q 13.3-q 14.1(74571645-77654540) \times 1 \\
5 q 14.3(90604937-92259476) \times 1 \\
5 q 15(93668778-95921236) \times 1 \\
9 p 23 p 22.3(14030668-16317401) \times 1 \\
\end{array}$ & $\begin{array}{l}2.4 \mathrm{Mb} \\
1.6 \mathrm{Mb} \\
1.9 \mathrm{Mb} \\
2.3 \mathrm{Mb}\end{array}$ & $\begin{array}{l}\text { loss } \\
\text { loss } \\
\text { loss } \\
\text { loss }\end{array}$ & de novo \\
\hline $21^{a}$ & F-2 & $\begin{array}{l}\text { facial dysmorphism; } \\
\text { short neck; soft, dry skin; } \\
\text { clinodactyly; bilateral } \\
\text { clubfoot, talipes echi- } \\
\text { novarus; hypoplastic } \\
\text { clitoris; tooth grinding; } \\
\text { multiple renal stones; } \\
\text { partial ACC; } \\
\text { normal MLPA result }\end{array}$ & $\begin{array}{l}46, X X, t(11 ; 13 ; 18)(13 p t e r \rightarrow \\
13 q 22:: 11 \text { p13 } \rightarrow 11 \text { pter; } \\
13 \text { qter } \rightarrow 13 \text { q22::11p13 } \rightarrow 11 \text { q24: } \\
: 18 q 21 \rightarrow 18 \text { qter; } 18 \text { pter } \rightarrow 18 q 21: \\
: 11 \text { q24 } \rightarrow 11 \text { qter })\end{array}$ & $\begin{array}{l}\text { 11p14.3(22454510-246887178)x1 } \\
\text { 18q21.2q21.32(48286848-58934492)x1 } \\
\text { 21q11.2q21.1(15598880-18332856)x1 }\end{array}$ & $\begin{array}{l}2.2 \mathrm{Mb} \\
10.6 \mathrm{Mb} \\
2.7 \mathrm{Mb}\end{array}$ & $\begin{array}{l}\text { loss } \\
\text { loss } \\
\text { loss }\end{array}$ & de novo \\
\hline
\end{tabular}

\#: case number; CMA: chromosomal microarray; PSMR: psychomotor retardation; MRI: magnetic resonance imaging; ASD: atrial septal defect; ID: intellectual disability; CHD: congenital heart defect; FISH: fluorescent in situ hybridization; MLPA: multiplex ligation-dependent probe amplification; ACC: agenesis of corpus callosum.

a De novo rearrangement.

${ }^{\mathrm{b}}$ Familial rearrangement. 
Table 2. Ultrasonography findings, karyotype and chromosomal microarray results for the prenatal patients.

\begin{tabular}{|c|c|c|c|c|c|c|c|}
\hline \# & $\begin{array}{l}\text { Weeks' Gestation/ } \\
\text { Invasive Procedure }\end{array}$ & $\begin{array}{c}\text { USG } \\
\text { Findings }\end{array}$ & $\begin{array}{c}\text { Initial } \\
\text { Karyotype }\end{array}$ & $\begin{array}{l}\text { CMA Result } \\
\text { (GRCh37/hg19] }\end{array}$ & Size & 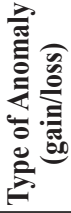 & 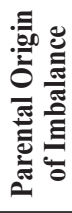 \\
\hline
\end{tabular}

\begin{tabular}{|c|c|c|c|c|c|c|c|}
\hline \multicolumn{8}{|c|}{ Translocations } \\
\hline $22^{\mathrm{a}}$ & $24 / \mathrm{CVS}$ & NT $(5 \mathrm{~mm})$ & $46, \mathrm{XX}, \mathrm{t}(2 ; 4)(\mathrm{p} 23 ; \mathrm{q} 31.1)$ & normal & - & - & - \\
\hline $23^{\mathrm{a}}$ & $21 / \mathrm{AC}$ & abdominal cysts; polyhydramnios & 46,XY,t(10;16)(q23.2;q13) & \begin{tabular}{|l|}
$10 q 23.1(86441275-$ \\
$87680071) \times 1$
\end{tabular} & $1.2 \mathrm{Mb}$ & loss & de novo \\
\hline $24^{\mathrm{a}}$ & $24 / \mathrm{AC}$ & dilated intestine; EIF & $46, \mathrm{XX}, \mathrm{t}(7 ; 12)(\mathrm{q} 36 ; \mathrm{q} 15)$ & normal & - & - & - \\
\hline $25^{\mathrm{a}}$ & $17 / \mathrm{AC}$ & CPCs; fetal intracranial cysts & $46, X Y, t(2 ; 3)(q 31.2 ; q 27.32)$ & normal & - & - & - \\
\hline $26^{\mathrm{a}}$ & $19 / \mathrm{AC}$ & CPCs & $46, \mathrm{XX}, \mathrm{t}(3 ; 16)(\mathrm{p} 21.3 \mathrm{q} 11)$ & \begin{tabular}{|l|} 
16p11.2(28220816- \\
$33816801) \times 3$
\end{tabular} & $5.5 \mathrm{Mb}$ & gain & maternal \\
\hline $27^{\mathrm{a}}$ & $23 / \mathrm{AC}$ & $\begin{array}{l}\text { SUA; HEB; increased cardio- } \\
\text { thoracic ration }\end{array}$ & $46, X Y, t(5 ; 13)(q 15 ; \mathrm{q} 22)$ & normal & - & - & - \\
\hline $28^{\mathrm{a}}$ & $20 / \mathrm{AC}$ & $\begin{array}{l}\text { bilateral ventriculomegaly; } \\
\text { hydrochephalus }\end{array}$ & $46, \mathrm{XX}, \mathrm{t}(8 ; 16)(\mathrm{q} 24.1 ; \mathrm{q} 12.1)$ & normal & - & - & - \\
\hline $29^{b}$ & $24 / \mathrm{AC}$ & asymmetric ventriculomegaly & 46,XY,t(15;17)(q24.1;q21.3)pat & normal & - & - & - \\
\hline $30^{\mathrm{b}}$ & 22/FBS & $\begin{array}{l}\text { bilateral pes equinovarus; } \\
\text { amniotic band sequence }\end{array}$ & 46,XY,t(11;15)(q13.3;q25)pat & normal & - & - & - \\
\hline
\end{tabular}

\begin{tabular}{|c|}
\hline Inversions \\
\hline
\end{tabular}

\begin{tabular}{|l|l|l|l|l|l|l|l|}
\hline $31^{\mathrm{b}}$ & $23 / \mathrm{FBS}$ & situs inversus totalis, CHD & $46, \mathrm{XY}, \operatorname{inv}(12)(\mathrm{p} 11.23 \mathrm{q} 15) \mathrm{mat}$ & normal & - & - & - \\
\hline $32^{\mathrm{b}}$ & $22 / \mathrm{AC}$ & $\begin{array}{l}\text { anhydramnios; laryngeal atresia; } \\
\text { bilateral renal agenesis; SUA; } \\
\text { absence/hypoplasia of lower } \\
\text { extremities; ambiguous genitalia }\end{array}$ & $46, \mathrm{XX}, \operatorname{inv}(7)(\mathrm{q} 11.2 \mathrm{q} 32) \mathrm{mat}$ & normal & - & - & - \\
\hline $33^{\mathrm{b}}$ & $24 / \mathrm{FBS}$ & $\begin{array}{l}\text { ascites; polyhydramnios; IUMF; } \\
\text { increased cardiothoracic ratio; } \\
\text { thick placenta }\end{array}$ & $46, \mathrm{XX}, \operatorname{inv}(6)(\mathrm{q} 21.2 \mathrm{q} 26)$ pat & normal & - & - & - \\
\hline
\end{tabular}

\begin{tabular}{|l|l|l|l|l|l|l|l|}
\hline \multicolumn{6}{|c|}{ Complex Chromosome Rearrangements } \\
\hline $34^{\mathrm{a}}$ & $23 /$ FBS & $\begin{array}{l}\text { IUGR; ambiguous genitalia; } \\
\text { micropenis; hand and foot } \\
\text { deformities; ASD }\end{array}$ & $\begin{array}{l}\text { 46,XY,t(1;9;11)(1pter } \rightarrow 1 \mathrm{q} 43: \\
\begin{array}{l}9 \mathrm{p} 22.2 \rightarrow 9 \mathrm{ppter} ; 1 \mathrm{pter} \rightarrow \\
11 \mathrm{p} 11.22:: 9 \mathrm{p} 21 \rightarrow 9 \mathrm{qter})\end{array}\end{array}$ & normal & - & - & - \\
\hline
\end{tabular}

USG: ultrasonography; CMA: chromosomal microarray; CVS: chorionic villus sampling; NT: nuchal translucency; AC: amniocentesis; EIF: echogenic intracardiac focus; CPCs: choroid plexus cysts; SUA: single umbilical artery; HEB: hyperechogenic bowel; FBS: fetal blood sampling; CHD: congenital heart failure; IUMF: in utero mort fetalis; IUGR: intrauterine growth restriction; ASD: autism spectrum disorder.

a De novo rearrangment.

${ }^{\mathrm{b}}$ Familial rearrangement.

(cases 23 and 26) (Table 2); both were de novo translocations. The imbalance rate for de novo cases was $25.0 \%$. One of the CNVs was a deletion $1.2 \mathrm{Mb}$ in size at the breakpoint of rearrangement. Another one was a duplication $5.5 \mathrm{Mb}$ in size on the same chromosome, but at different bands. This $\mathrm{CNV}$ was inherited from a healthy mother.

\section{DISCUSSION}

Molecular karyotyping allowed us to detect genomewide chromosomal imbalances even in size of kbs using
DNA copy number and/or SNP variation probes [10]. Recent reports have suggested that ABCRs in patients having abnormal phenotypes can be more complex at the molecular level than suspected by karyotyping. Therefore, ABCRs either de novo or familial, should be investigated at the molecular level, if the phenotype is affected [5,11-15].

Molecular karyotyping reveals the breakpoints of the rearrangements, especially for inversions and insertions, more precisely than the karyotyping seen in our cases 10 , 14, and 21. As CMA is a genome-wide technique, it allowed us to detect the imbalances elsewhere in the genome 
Table 3. Comparison with the previous reports for postnatal de novo patients.

\begin{tabular}{|c|c|c|c|c|c|c|c|}
\hline Study & Array Platform & Translocation & Inversion & CCRs & Total & $\begin{array}{l}\text { Imbalances } \\
\text { at the } \\
\text { Breakpoint }\end{array}$ & $\begin{array}{c}\text { Imbalances } \\
\text { at Different } \\
\text { Breakpoint } \\
\text { Regions }\end{array}$ \\
\hline [13] & $1 \mathrm{Mb} \mathrm{BAC}$ & $5 / 8(62.5)$ & - & $0 / 2(0.0)$ & $5 / 10(50.0)$ & $2 / 10(20.0)$ & $3 / 10(30.0)$ \\
\hline [11] & Agilent $44 \mathrm{~K}$ or $244 \mathrm{k}$ & $11 / 27(40.0)$ & - & $12 / 13(92.3)$ & $23 / 40(57.5)$ & $16 / 40(40.0)$ & $7 / 40(17.5)$ \\
\hline [37] & $\begin{array}{l}\text { Agilent } 244 \mathrm{k} / 2600, \mathrm{BAC}, \text { Spectral } \\
\text { Genomics }\end{array}$ & 4/9 (44.4) & - & - & 4/9 (44.4) & 1/9 (11.1) & $3 / 9(33.3)$ \\
\hline$[34]^{\mathrm{a}}$ & Whole Genome Tilepath 30k & $4 / 11(36.3)$ & - & $1 / 2(50.0)$ & $5 / 13(38.4)$ & $4 / 13(30.7)$ & $1 / 13(7.7)$ \\
\hline [5] & Cytochip Bluegnome $1 \mathrm{Mb}$ & $2 / 6(33.2)$ & - & - & $2 / 6(33.2)$ & $1 / 6(16.6)$ & $1 / 6(16.6)$ \\
\hline [14] & Agilent $44 \mathrm{~K}$ or $244 \mathrm{k}$ & $8 / 21(38.1)$ & $4 / 7(57.1)$ & $4 / 5(80.0)$ & $16 / 33(48.5)$ & $9 / 33(27.3)$ & $7 / 33(21.2)$ \\
\hline$[16]^{\mathrm{a}}$ & Affymetrix GeneChip 250k & $3 / 3(100.0)$ & $2 / 2(100.0)$ & - & $5 / 5(100.0)$ & $2 / 5(40.0)$ & $3 / 5(60.0)$ \\
\hline$[12]^{\mathrm{a}}$ & $\begin{array}{l}\text { 32k BAC, } 105 \mathrm{k} / 244 \mathrm{k} \text { Agilent, } \\
\text { 250k Affymetrix }\end{array}$ & $11 / 40(27.5)$ & $0 / 6(0.0)$ & $7 / 8(87.5)$ & $18 / 54(33.3)$ & $11 / 54(20.3)$ & $7 / 54(13.0)$ \\
\hline$[15]^{\mathrm{a}}$ & 370k, 370-Duo Illumina & $2 / 5(40.0)$ & $2 / 4(50.0)$ & - & 4/9 (44.4) & $2 / 9(22.2)$ & $2 / 9(22.2)$ \\
\hline [17] & 135k NimbleGen CGX-3 & $3 / 7(43.0)$ & $1 / 4(25.0)$ & - & 4/11 (36.4) & $3 / 11(27.3)$ & $1 / 11(9.1)$ \\
\hline$[38]$ & SNP 6.0/750k Affymetrix & $1 / 11(9.0)$ & $0 / 3(0.0)$ & $1 / 1(100.0)$ & $3 / 15(20.0)$ & $1 / 15(6.7)$ & $2 / 15(13.3)$ \\
\hline This study & 1.4M NimbleGen CGX-3/ 180k Agilent & $1 / 5(20.0)$ & $1 / 1(100.0)$ & $5 / 8(62.5)$ & $7 / 14(50.0)$ & $2 / 14(14.3)$ & $5 / 14(35.7)$ \\
\hline Total & & $55 / 153(35.9)$ & $10 / 27(37.0)$ & $29 / 39(74.4)$ & $96 / 219(43.8)$ & $54 / 219(24.6)$ & $42 / 219(19.2)$ \\
\hline
\end{tabular}

CCRs: complex chromosome rearrangements; BAC: bacterial artificial chromosome.

Manufacturers' locations. BAC: in-house methods; Agilent Technologies Inc.: Santa Clara, CA, USA; Spectral Genomics Co.: Houston, TX, USA; Whole Genome Tilepath: in-house methods; BlueGnome Ltd.: Cambridge, Cambridgeshire, UK; Affymetrix, Inc.: Santa Clara, CA, USA; Illumina Inc.: San Diego, CA, USA; Roche NimbleGen, Inc.: Madison, WI, USA.

${ }^{a}$ Unknown parental origins for karyotype result of the reported cases were excluded.

such as in our cases 15, 16, 20, and 21. Eight CNVs at different locations, apart from the suspected breakpoints, indicates the complexity of the CCRs and effectiveness of CMA [16].

No molecular imbalances were observed in our familial ABCRs (five prenatal, seven postnatal). However, Gijsbers et al. [16] reported a deletion at the unrelated chromosome in one case of four familial translocation cases (25.0\%). Sismani et al. [5] determined two CNVs at the translocation breakpoints in one out of the six familial translocations (16.6\%). In another study from SchluthBolard et al. [14] containing 14 familial cases (seven translocations, five inversions, two CCRs), imbalance rates were $14.3 \%$ for translocations, $40.0 \%$ for inversions and $50.0 \%$ for CCR and one inversion case had two different $\mathrm{CNVs}$, one at the breakpoint and the other one at an independent region. Tabet et al. [15] reported four familial translocation and three inversion cases, of which one had a deletion at the related region of the inversion (14.3\%). Sezin et al. [17] reported four familial cases with no cryptic imbalance. To sum up, the CNV rate in familial cases was $14.9 \%$ in a total of 47 cases. Six of eight imbalanced cases were at the breakpoints $(75.0 \%)$, whereas the remaining two were at unrelated locations $(25.0 \%)$. These results show that the risk for imbalances is higher for inversions and CCRs than translocations, and familial ABCRs can also have imbalances at the breakpoints or somewhere in the genome, coincidentally or not. If aCGH reveals no imbalances in cases presenting with phenotypical findings, they should be further investigated for monogenic disorders [18]. In our postnatal series, six of the seven familial ABCR carriers had a consanguineous marriage, which enhances the possibility of monogenic disorders.

The rate of de novo imbalances detected by CMA was higher in postnatal than prenatal cases (50.0 vs. $25.0 \%)$ in this study, because postnatal cases were preselected due to their distinct abnormal phenotype. In our center, CMA was offered in the presence of pathological fetal ultrasound findings after normal fetal karyotypes, but also in cases with de novo ABCRs, even if ultrasound findings were normal. A prenatal case presented with polyhydramnios and abdominal cysts had a $1.2 \mathrm{Mb}$ deletion containing GRID1, Mir_544, AK097624,LOC100507470, AX746544, 7SK genes at the 10q23.1 band. Van Bon et al. [19] reported 12 patients having a deletion on 10q22.3-q23.3. One patient had an atrioventricular septal defect (AVSD), dysmorphic features, diaphragmatic eventration and undescended testes, and CMA revealed a small interstitial GRID1 deletion comprising exons 5 to $8,0.2 \mathrm{Mb}$ in size. The GRIDl gene has been also reported to be associated with schizophrenia 
$[20,21]$. Because the ultrasound findings of our case was not compatible with the cases of Van Bon et al. [19], and deletions of this region have not yet been associated with a particular phenotype, this deletion was interpreted as variations of unknown significance (VUS). In another case, presenting with only choroid plexus cysts, a $5.5 \mathrm{Mb}$ duplication was inherited from the healthy mother. Surprisingly, prenatal cases presenting with major structural anomalies (cases 31, 32, 33 and 34) had no imbalances by CMA, and further studies to search for monogenic disorders were planned. De Gregori et al. [11] also reported 14 de novo prenatal translocations including one presenting with abnormal USG findings, one had normal USG but delayed psychomotor development at 6 months of age and none of them had an imbalance by CMA. Evangelidou et al. [22] reported one familial and four de novo translocation cases with abnormal USG findings; all cases were also found to be normal by CMA. Due to the limited number of published cases, experiences with prenatal cases are not sufficient to estimate a risk figure for prenatal ABCRs with abnormal USG findings. When de novo ABCRs detected by fetal karyotyping, independent from the phenotypical findings, molecular karyotyping should be applied, because clinical findings are limited with only ultrasound examination.

Variations of unknown significance detected in prenatal CMA studies are confusing and genetic counseling is difficult. Therefore, to decrease the VUS possibility, the standards and guidelines of 2013 [9] recommends reporting the variations that are $>400 \mathrm{~kb}$ for both deletions and duplications in prenatal and postnatal studies by whole genome array platforms. Different countries have their approach for prenatal cases, such as including genes number [ $>18$ genes (Belgium), size of imbalances $(>500 \mathrm{~kb}$ for deletions and $>1.0 \mathrm{Mb}$ for duplications (Canada)], choosing array platforms reducing densities of the probes [23-25]. In this study, we initially used higher CMA resolution $(1.4 \mathrm{Mb})$ for both postnatal and prenatal cases. Detected imbalances in prenatal cases were over $1.0 \mathrm{Mb}$, which could be confirmed by $180 \mathrm{~K}$ resolution. In some cases, a lower resolution can cause difficulties to search the whole genome or the size of the CNV may differ from the actual size due to the limited number of probes [26].

Parental array studies showed that the detected duplication at $16 \mathrm{p} 11$ in case 26 was inherited maternally, which, coincidentally, was on one of the ABCR-involved chromosomes. Imbalances (size range $579 \mathrm{~kb}$ to $4.6 \mathrm{Mb}$ ) including deletions and duplications at $16 \mathrm{p} 11$ showing incomplete penetrance/variable expressivity, can be associated with global development delay, behavioral problems, epilepsy, autism [27]. The critical region comprising breakpoints four and five (BP4-BP5) (600 kb, chr16; 29.6-30.2 mb-
HG19) called 16p11.2 microdeletion/microduplication syndrome is reported in about three in 10,000 [28]. Most of the $16 \mathrm{p} 11.2$ microduplications $(70.0 \%)$ are familial, and the clinical findings are variable from severe to mild [29]. The size of microduplication in our case was larger than the critical region, the healthy carrier mother decided to continue the pregnancy. Clinical evaluations of the newborn revealed normal results, clinical follow-up controls of the baby were planned. In general, the detection of duplications is troublesome, either cytogenetically or clinically due to the nonspecific and variable phenotypes. Based on these experiences, it was expected that the frequency of the duplications is higher by CMA than in karyotyping in patients with behavioral problems or autism without distinct dysmorphic features [30]. Therefore, genetic counseling for de novo duplications detected prenatally is still challenging.

When the data of published postnatal de novo series are combined, $35.9 \%$ of the apparently balanced de novo translocations were, in fact, unbalanced. This rate was $20.0 \%$ in our series (Table 3 ). Imbalance rate in inversions was as much as in translocations (37.0\%). There was only one inversion patient in our series and she had a deletion. As expected, the highest submicroscopic imbalance rate (74.4\%) was observed in CCRs. In our series, it was $62.5 \%$. The difference $(\sim 12.0 \%)$ could be explained by the presence of two Xp21-autosome translocation cases in our series. Both cases manifested female carriers of Duchenne muscular dystrophy (DMD) had also been investigated by multiplex ligation-dependent probe amplification (MLPA) (MRC-Holland, Amsterdam, The Netherlands) and by next generation sequencing (NGS) techniques, and no mutations were detected. The causing factor was not imbalances at the molecular level, but the possibly skewed X inactivation, where the $\mathrm{X}$ chromosome carrying the normal dystrophin gene is preferentially inactivated to save the translocated autosomal segment on the derivative $\mathrm{X}$ chromosome [31]. If these two cases were excluded, the imbalance rate would be $83.3 \%$ in our series.

The imbalances at unrelated regions of the breakpoints/chromosomes observed in five CCR cases (cases 10, $15,16,20,21)$ demonstrate the advantage of genome-wide array studies. Multiple breaks and consecutive micro-deletions in these cases show the complexity of the CCRs, and support that de novo CCRs occur due to the multiple breaks in the genome and increased genomic instability. The term 'chromothripsis' is used to describe 'chromosome shattering,' which means chromosomes are first fragmented into many pieces and then the fragments stick back together randomly due to the DNA repair processes [32]. Analysis of CCRs in patients with congenital disorders showed that chromothripsis is not applicable for all complex germline 
rearrangements. The term 'chromosoanasynthesis,' which means repeated chromosome synthesis was suggested by Liu et al. [33] for describing multiple template switch events that may occur during the germline CCRs formation process. Many more CCRs should be investigated by whole-genome analysis to increase the knowledge and understanding the underlying occurrence mechanism of the CCRs [34-36].

Altogether, the imbalance rate of de novo ABCRs is $24.6 \%$ at the breakpoints and $19.2 \%$ at different regions apart from the breakpoints. These rates can be used in genetic counseling related to de novo ABCRs.

In conclusion: 1) the current study supports previous studies, that the cryptic genomic imbalances are high $(43.8 \%)$ in patients of de novo ABCRs with abnormal phenotype. 2) Patients presenting with multiple congenital anomalies, intellectual disability, and carrying de novo or familial ABCRs, whatever the type of rearrangement (translocation, inversion, or CCR), should be studied systematically by CMA. 3) Chromosomal microarray investigation is more important in cases with de novo ABCRs detected prenatally, as the results are essential for genetic counseling and decision-making process of the parents. Studies focused on prenatal ABCR carriers with abnormal ultrasound findings are limited and no submicroscopic imbalance was reported. Abnormality rate detected by CMA was $25.0 \%$ in our de novo prenatal cohort. 4) Chromosomal microarray promises to detect genome-wide imbalances at the kb level. However, further studies are needed for other mechanisms such as gene fusions or gene disruptions that might explain the phenotype of affected ABCR carriers. All unexplained patients should be examined for single gene disorders.

Declaration of Interest. The authors report no conflicts of interest. The authors alone are responsible for the content and writing of this article.

Funding. This study was supported by the Istanbul University Scientific Research Project Unit [Project Nos: 34325, 8563 and 25099].

\section{REFERENCES}

1. Jacobs PA, Browne C, Gregson N, Joyce C, White $\mathrm{H}$. Estimates of the frequency of chromosome abnormalities detectable in unselected newborns using moderate levels of banding. J Med Genet. 1992; 29(2): 103-108.

2. Kirchhoff M, Rose H, Lundsteen C. High resolution comparative genomic hybridisation in clinical cytogenetics. J Med Genet. 2001; 38(11): 740-744.
3. Warburton D. De novo balanced chromosome rearrangements and extra marker chromosomes identified at prenatal diagnosis: Clinical significance and distribution of breakpoints. Am J Hum Genet. 1991; 49(5): 995-1013.

4. Madan K, Nieuwint AW, van Bever Y. Recombination in a balanced complex translocation of a mother leading to a balanced reciprocal translocation in the child. Review of 60 cases of balanced complex translocations. Hum Genet. 1997; 99(6): 806-815.

5. Sismani C, Kitsiou-Tzeli S, Ioannides M, Christodoulou C, Anastasiadou V, Stylianidou G, et al. Cryptic genomic imbalances in patients with de novo or familial apparently balanced translocations and abnormal phenotype. Mol Cytogenet. 2008; 1(7): 15-23.

6. Menten B, Maas N, Thienpont B, Buysse K, Vandesompele J, Melotte C, et al. Emerging patterns of cryptic chromosomal imbalance in patients with idiopathic mental retardation and multiple congenital anomalies: A new series of 140 patients and review of published reports. J Med Genet. 2006; 43(8): 625-633.

7. Rauch A, Rüschendorf F, Huang J, Trautmann U, Becker C, Thiel C, et al. Molecular karyotyping using an SNP array for genomewide genotyping. J Med Genet. 2004; 41(12): 916-922.

8. Riggs ER, Andersen EF, Cherry AM, Kantarci S, Kearney H, Patel A, et al. Technical standards for the interpretation and reporting of constitutional copynumber variants: A joint consensus recommendation of the American College of Medical Genetics and Genomics (ACMG) and the Clinical Genome Resource (ClinGen). Genet Med. 2020; 22(2): 245-257.

9. South ST, Lee C, Lamb AN, Higgins AW, Kearney HM; Working Group for the American College of Medical Genetics and Genomics Laboratory Quality Assuarance Committee. ACMG Standards and Guidelines for constitutional cytogenomic microarray analysis, including postnatal and prenatal applications: Revision 2013. Genet Med. 2013; 15(11): 901909 (https://pubmed.ncbi.nlm. nih.gov/2407193/).

10. Darilek S, Ward P, Pursley A, Plunkett K, Furman P, Magoulas $\mathrm{P}$, et al. Pre- and postnatal genetic testing by array-comparative genomic hybridization: Genetic counseling perspectives. Genet Med. 2008; 10(1): 13-18.

11. De Gregori M, Ciccone R, Magini P, Pramparo T, Gimelli S, Messa J, et al. Cryptic deletions are a common finding in "balanced" reciprocal and complex chromosome rearrangements: A study of 59 patients. J Med Genet. 2007; 44(12): 750-762. 
12. Feenstra I, Hanemaaijer N, Sikkema-Raddatz B, Yntema H, Dijkhuizen T, Lugtenberg D, et al. Balanced into array: genome-wide array analysis in 54 patients with an apparently balanced de novo chromosome rearrangement and a meta-analysis. Eur J Hum Genet. 2011; 19(11): 1152-1160.

13. Gribble SM, Prigmore E, Burford DC, Porter KM, Ng $\mathrm{BL}$, Douglas EJ, et al. The complex nature of constitutional de novo apparently balanced translocations in patients presenting with abnormal phenotypes. $\mathrm{J}$ Med Genet. 2005; 42(1): 8-16.

14. Schluth-Bolard C, Delobel B, Sanlaville D, Boute O, Cuisset JM, Sukno S, et al. Cryptic genomic imbalances in de novo and inherited apparently balanced chromosomal rearrangements: Array CGH study of 47 unrelated cases. Eur J Med Genet. 2009; 52(5): 291-296.

15. Tabet AC, Verloes A, Pilorge M, Delaby E, Delorme $\mathrm{R}$, Nygren $\mathrm{G}$, et al. Complex nature of apparently balanced chromosomal rearrangements in patients with autism spectrum disorder. Mol Autism. 2015; 6(5): 19-32.

16. Gijsbers AC, Bosch CA, Dauwerse JG, Giromus O, Hansson K, Hilhorst-Hofstee Y, et al. Additional cryptic CNVs in mentally retarded patients with apparently balanced karyotypes. Eur J Med Genet. 2010; 53(5): 227-233.

17. Yakut S, Cetin Z, Clark OA, Nur BG, Mihci E, Karauzum SB. Associations between the clinical findings of cases having submicroscopic chromosomal imbalances at chromosomal breakpoints of apparently balanced structural rearrangements. Gene Rep. 2017; 7(7): 50-58 (https:// www.sciencedirect.com/science/ article/pii/S245201441 7300092).

18. Dong Z, Wang H, Chen H, Jiang H, Yuan J, Yang Z, et al. Identification of balanced chromosomal rearrangements previously unknown among participants in the 1000 Genomes Project: Implications for interpretation of structural variation in genomes and the future of clinical cytogenetics. Genet Med. 2018; 20(7): 697-707.

19. van Bon BW, Balciuniene J, Fruhman G, Naga-mani SC, Broome DL, Cameron E, et al. The phenotype of recurrent 10q22q23 deletions and duplications. Eur J Hum Genet. 2011; 19(4): 400-408.

20. Nenadic I, Maitra R, Scherpiet S, Gaser C, Schultz CC, Schachtzabel C, et al. Glutamate receptor $\delta 1$ (GRID1) genetic variation and brain structure in schizophrenia. J Psychiat Res. 2012; 46(12): 15311539.
21. Treutlein J, Mühleisen TW, Frank J, Mattheisen M, Herms S, Ludwig KU, et al. Dissection of phenotype reveals possible association between schizophrenia and Glutamate Receptor Delta 1 (GRID1) gene promoter. J Psychiatr Res. 2009; 111(1-3): 123-130 (https://pubmed. ncbi.nlm.nih.gov/19346103/).

22. Evangelidou P, Sismani C, Ioannides M, Christodoulou C, Koumbaris G, Kallikas I, et al. Clinical application of whole-genome array CGH during prenatal diagnosis: Study of 25 selected pregnancies with abnormal ultrasound findings or apparently balanced structural aberrations. Mol Cytogenet. 2010; 3: 24.

23. Armour CM, Dougan SD, Brock JA, Chari R, Chodirker BN, DeBie I, et al. Practice guideline: Joint CCMG-SOGC recommendations for the use of chromosomal microarray analysis for prenatal diagnosis and assessment of fetal loss in Canada. J Med Genet. 2018; 55(4): 215-221.

24. Shaffer LG, Dabell MP, Rosenfeld JA, Neill NJ, Ballif $\mathrm{BC}$, Coppinger J, et al. Referral patterns for microarray testing in prenatal diagnosis. Prenat Diagn. 2012; 32(4): 344-350.

25. Vanakker O, Vilain C, Janssens K, Van der Aa N, Smits $\mathrm{G}$, Bandelier $\mathrm{C}$, et al. Implementation of genomic arrays in prenatal diagnosis: The Belgian approach to meet the challenges. Eur J Med Genet. 2014; 57(4): 151-156.

26. Levy B, Burnside RD. Are all chromosome microarrays the same? What clinicians need to know. Prenat Diagn. 2019; 39(3): 157-164.

27. Shinawi M, Liu P, Kang S-HL, Shen J, Belmont JW, Scott DA, et al. Recurrent reciprocal 16p11.2 rearrangements associated with global developmental delay, behavioural problems, dysmorphism, epilepsy, and abnormal head size. J Med Genet. 2010; 47(5): 332-341.

28. Weiss LA, Shen Y, Korn JM, Arking DE, Miller DT, Fossdal R, et al. Association between microdeletion and microduplication at $16 \mathrm{p} 11.2$ and autism. N Engl J Med. 2008; 358(7): 667-675.

29. D'Angelo D, Lebon S, Chen Q, Martin-Brevet S, Snyder LG, Hippolyte L, et al.; Cardiff University Experiences of Children with Copy Number Variants (ECHO) Study; 16p11.2 European Consortium; Simons Variation in Individuals Project (VIP) Consortium. Defining the effect of the 16p11.2 duplication on cognition, behavior, and medical comorbidities. JAMA Psychiatry. 2016; 73(1): 20-30.

30. Shinawi M, Cheung SW. The array CGH and its clinical applications. Drug Discov Today. 2008; 13(1718): 760-770. 
31. Trippe H, Wieczorek S, Kötting J, Kress W, Schara U. Xp21/A translocation: A rarely considered genetic cause for manifesting carriers of duchenne muscular dystrophy. Neuropediatrics. 2014; 45(5): 333-335.

32. Hatch EM, Hetzer MW. Chromothripsis. Curr Biol. 2015; 25(10): R397-R399.

33. Liu P, Carvalho CMB, Hastings PJ, Lupski JR. Mechanisms for recurrent and complex human genomic rearrangements. Curr Opin Genet Dev. 2012; 22(3): 211-220.

34. Baptista J, Mercer C, Prigmore E, Gribble SM, Carter NP, Maloney V, et al. Breakpoint mapping and array $\mathrm{CGH}$ in translocations: Comparison of a phenotypically normal and an abnormal cohort. Am J Hum Genet. 2008; 82(4): 927-936.

35. Chen W, Ullmann R, Langnick C, Menzel C, Wotschofsky Z, Hu H, et al. Breakpoint analysis of balanced chromosome rearrangements by nextgeneration paired-end sequencing. Eur J Hum Genet. 2010; 18(5): 539-543.
36. Talkowski ME, Ernst C, Heilbut A, Chiang C, Hanscom C, Lindgren A, et al. Next-generation sequencing strategies enable routine detection of balanced chromosome rearrangements for clinical diagnostics and genetic research. Am J Hum Genet. 2011; 88(4): 469-481.

37. Higgins AW, Alkuraya FS, Bosco AF, Brown KK, Bruns GA, Donovan DJ, et al. Characterization of apparently balanced chromosomal rearrangements from the developmental genome anatomy project. Am J Hum Genet. 2008; 82(3): 712-722.

38. Simioni M, Artiguenave F, Meyer V, Sgardioli IC, Viguetti-Campos NL, Lopes Monlleó I, et al. Genomic investigation of balanced chromosomal rearrangements in patients with abnormal phenotypes. Mol Syndromol. 2017; 8(4): 187-194. 